\title{
Correction to: The Photosynthetic Adventure of Paulinella Spp
}

Przemysław Gagat, Katarzyna Sidorczuk, Filip Pietluch, and Pawel Mackiewicz

\section{Correction to:}

Chapter 13 in: M. Kloc (ed.), Symbiosis: Cellular, Molecular, Medical and Evolutionary Aspects, https://doi.org/10.1007/978-3-030-51849-3_13

The funder information was missing from the acknowledgement section and the funding agency has asked the author to change this. The acknowledgement should read as:

Acknowledgments We are grateful to the reviewers for their helpful comments. We also would like to thank Dr. Malgorzata Kloc for inviting us to write this chapter. This work was supported by the National Science Centre grant UMO-2017/26/D/ NZ8/00444 to P. Gagat and UMO-2018/31/N/NZ2/01338 to K. Sidorczuk.

The original chapter has been corrected. 